Brief note

\title{
MECHANISM OF A MOTION PLATFORM INDUCING THE MOVEMENT OF THE OPERATOR'S SEAT IN A HEAVY MACHINE
}

\author{
G. TORA and W. TRZASKA* \\ Institute of Machine Design, Faculty of Mechanical Engineering \\ Cracow University of Technology \\ al. Jana Pawła II 37, 31-864 Krakow, POLAND \\ E-mails: tora@mech.pk.edu.pl; wtrzaska@mech.pk.edu.pl
}

\begin{abstract}
One of the major work safety issues involves the investigations of machine vibrations and the way they affect the machine operators. These investigations can be performed in the laboratory conditions in a setup in which the seat vibrations are induced by a motion platform. A complex mechanism is proposed implementing 3 translations and 2 rotation movements, and actuated by three electric motors. This study investigates the design structure of the real motion platform mechanism and provides the synthesis of link dimensioning. Kinematic dependencies are formulated and the Jacobian matrix is derived accordingly to solve the equations of motion. Computer simulation procedure yields the critical seat vibration frequency and amplitudes for the available asynchronous motors.
\end{abstract}

Key words: simulator, synthesis, modeling of the mechanism.

\section{Introduction}

A majority of wheeled machines operated nowadays are operator-controlled. In accordance with the applicable regulations [1], ensuring the required work safety for the machine operators and the personnel working in the vicinity of a heavy machine implicates a number of requirements to be satisfied, these requirements are often treated as single specification limits. Improving the operator's work safety is perceived as enhancing the operators' comfort at work to prevent their tiredness and enhance concentration. The direct effect of these actions is increased efficiency and work safety, at the same time the machine's performance and its work quality are improved. Relevant safety requirements include the arrangement of the cab's interior, an adequate field of vision from the operator's seat, proper lighting, control gear configuration, ensuring the right microclimate inside the cab, compliance with the applicable vibration and noise control standards, and others. Continuing efforts made to improve the work safety of heavy machine operators have prompted the research work aimed to determine the effects of particular factors adversely affecting the operator's well being. Investigations can be conducted on the site, on real machines, and recently motion simulators emulating the real operating conditions have come into use. The application of simulators helps reduce the costs of testing procedures, allowing them to be repeated under the same conditions. Another advantage of a simulator is that the most adverse operating conditions and combinations of loads acting upon the machine and the operator and the emergency conditions can be handled as well [2], [3]. Conducting the tests in controlled conditions improves the safety level during the tests, reducing the time required for experimenting, which is of particular importance when machines are to be designed concurrently. Furthermore, simulators enable safe training of machine operators. Other applications of motion simulators includes the tests of drivers' performance under the influence of alcohol and drugs [4], [5] and the ways drivers respond to new

\footnotetext{
* To whom correspondence should be addressed
} 
drugs. Such tests cannot be performed on public roads because of the traffic regulations and the potential risk to the remaining road users [6].

This study is focused on the mechanical component of the system referred to as a motion platform, emulating the movements of the operator's seat in a mobile heavy machine, for example a wheeled loader. The mobile platform ought to implement a predetermined sequence of motions, the sequence being obtained from measurements taken on a real machine.

\section{Structure of a motion platform mechanism inducing the motion of an operator's seat in a heavy machine}

Research efforts to find the structure of a spatial mechanism to be used in kinematic systems in parallel manipulators are summarised in works [7], [8], [9]. Modelling of the platform simulator is considered in [10] whilst a thorough analysis of solutions and classification of simulators is provided in [6].

The design structure of the motion platform mechanism considered in the study is shown in Fig.1. It should enable the seat's motion in five DOFs, excluding the rotating motion around the vertical axis. It is proposed that the seat motions should be implemented in the longitudinal and lateral vertical planes using two four-bar linkage mechanisms whilst the Sarrus mechanism is used to effect the seat motion in the direction normal to the upper platform.

Seat movements are caused by, inter alia, the machine ride and its tilting due to rough terrain. Its instantaneous centre of gravity (cog), associated with the tilting motion, is on the road level [9]. When the dimensions of the four-bar links are selected properly, the instantaneous rotation centre of the platform with the seat is found near the horizontal plane whose position corresponds to the road surface and hence the tilting motion of the machine can be reproduced in laboratory conditions, although the procedure may involve a certain error. Four-bar linkages in the moving platform mechanism are set in motion by two drives, positioned on an intermediate mobile platform, which induce linear motion in the longitudinal and lateral direction, and rotating motions around the longitudinal and lateral direction, correlated with linear movement. Certain assumptions were made underlying the calculations of the motion platform mechanism dimensions and dependencies which are of key importance from the standpoint of kinematics:

- the instantaneous rotation centre of the platform with the seat in its middle position is located at the distance equal to the distance between the base point of the seat (SIP) cushion and the road surface,

- four-bar linkages are symmetrical and should not reach the extreme positions in the projected operating ranges;

The Sarrus mechanism responsible for rectilinear guiding of the seat is equipped with two springs attached to mobile axes of the joints in the links. The function of these springs is to relieve the drive lifting the seat of the load due to the operator's and seat's gravity force.

The proposed structure of the moving platform mechanism has two advantages:

- it allows an approximate reproduction of the seat motions in five DOFs using three drives,

- the seat lifting drive is to a large extent relieved of the load due to the gravity force of the operator, the seat and the moving platform.

The main purpose of the present study is to test whether the moving platform is capable of implementing the predetermined motion and to establish the dependency between its operating range and the parameters of asynchronous motors. 


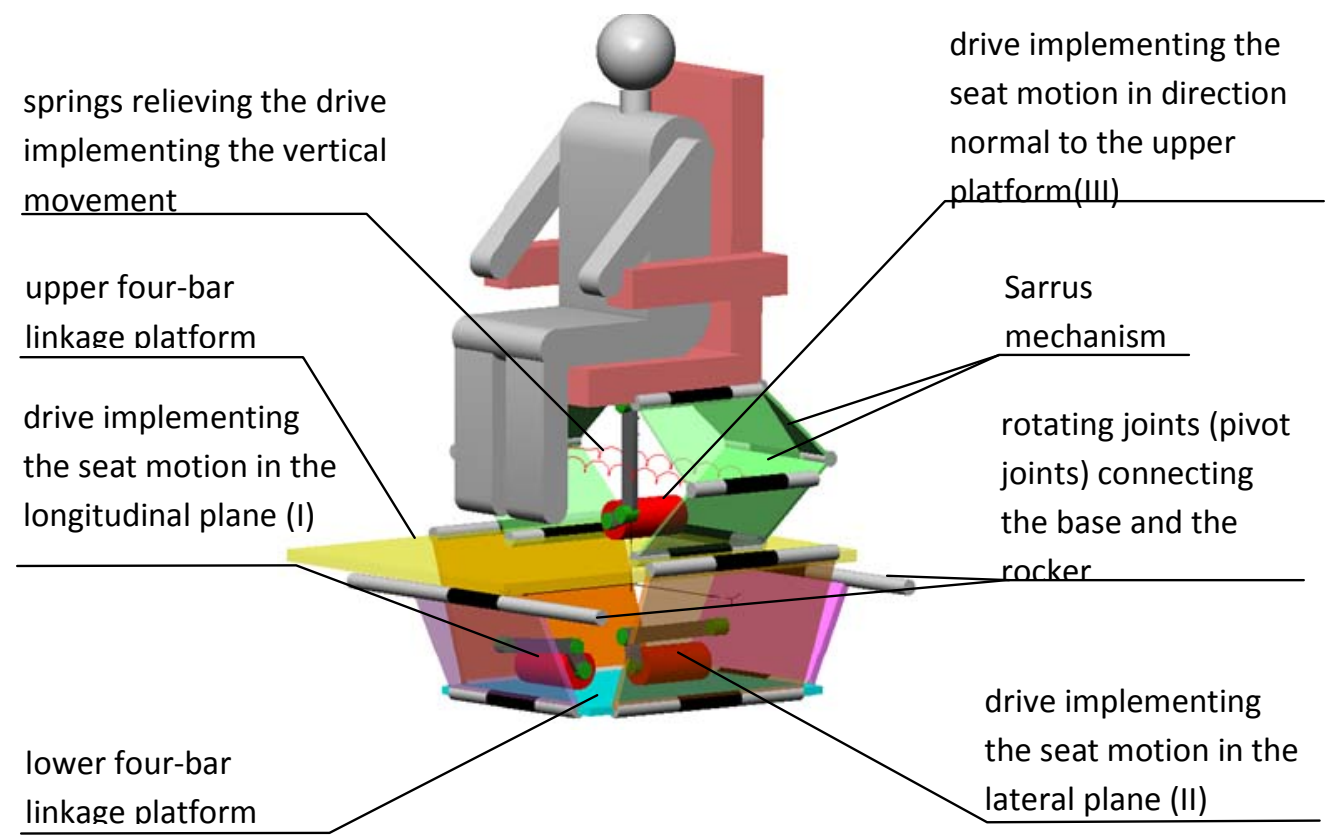

Fig.1. Structure of the motion platform mechanism.

\section{Kinematic and dynamic model of the motion platform mechanism}

The kinematic analysis uses versors [9], [11] which determine the orientation of the mechanism links. Figure 2 shows an equivalent kinematic diagram of the motion platform mechanism minus three drives, the crank systems and the Sarrus mechanism. An analysis of links' orientation consists in finding versors related to them by the function of fixed versors of the base: $\hat{\boldsymbol{x}}_{0}, \hat{\boldsymbol{y}}_{0}, \hat{\boldsymbol{z}}_{0}$ and mobile versors $\hat{\boldsymbol{q}}_{1}, \hat{\boldsymbol{q}}_{4}$, whose orientation is determined by the drives.

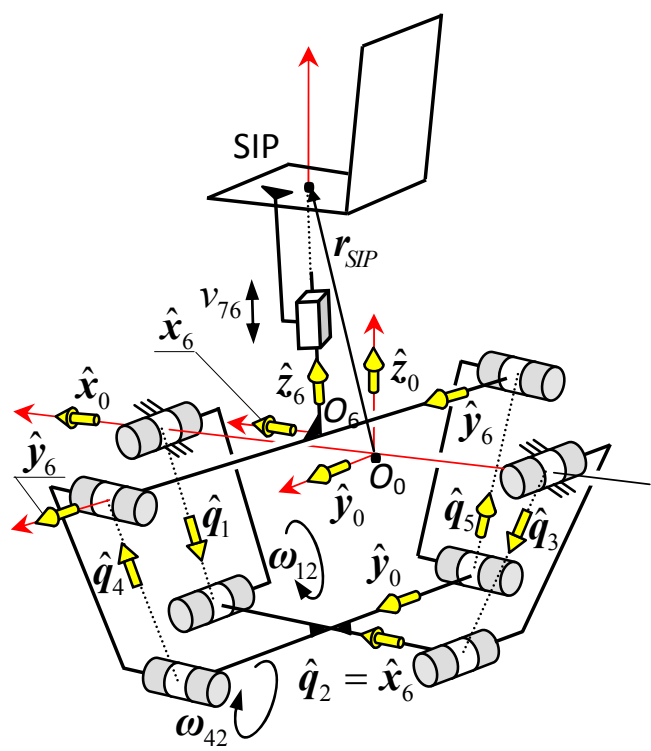

Fig.2. Equivalent kinematic diagram of the real motion platform mechanism. 
Equations of vector polygons, of the lower four-bar linkage connected to the base and of the upper linkage, yield all required versors

$$
\left\{\begin{array}{l}
l_{2} \hat{\boldsymbol{q}}_{2}+l_{3} \hat{\boldsymbol{q}}_{3}=l_{1} \hat{\boldsymbol{q}}_{1}+l_{0} \hat{\boldsymbol{x}}_{0}, \\
l_{5} \hat{\boldsymbol{q}}_{5}+l_{p} \hat{\boldsymbol{y}}_{p}=l_{4} \hat{\boldsymbol{q}}_{4}+l_{2} \hat{\boldsymbol{y}}_{0}
\end{array}\right.
$$

where: $\hat{\boldsymbol{q}}_{i}$ - the $i$ link versor, $l_{0}, l_{1}, l_{2}, l_{2^{\prime}}, l_{3}, l_{4}, l_{5}, l_{6}$ - the assumed link dimensions corresponding to particular versors (versor index and the corresponding dimension is the same as the link index, for the link 2 two indices are given: 2 and 2' to distinguish two different dimensions).

A solution to the system of Eqs (3.1) can be written in a generalised form

$$
\hat{\boldsymbol{q}}_{i}=f_{i}\left(\hat{\boldsymbol{x}}_{0}, \hat{\boldsymbol{y}}_{0}, \hat{\boldsymbol{z}}_{0}, \hat{\boldsymbol{q}}_{1}, \hat{\boldsymbol{q}}_{4}\right) \quad i=2,3,5 .
$$

Recalling Fig.2, versors expressing the orientation of platform 6 and the seat become

$$
\left\{\begin{array}{l}
\hat{\boldsymbol{x}}_{6}=k_{x x} \hat{\boldsymbol{x}}_{0}+k_{x z} \hat{\boldsymbol{z}}_{0}, \\
\hat{\boldsymbol{y}}_{6}=k_{y x} \hat{\boldsymbol{x}}_{0}+k_{y y} \hat{\boldsymbol{y}}_{0}+k_{y z} \hat{\boldsymbol{z}}_{0}, \\
\hat{\boldsymbol{z}}_{6}=\hat{\boldsymbol{x}}_{6} \times \hat{\boldsymbol{y}}_{6}
\end{array}\right.
$$

where: $k_{x x}, k_{x z}, k_{y x}, k_{y y}, k_{y z}$ - are coefficients derived from the system of Eqs (3.1).

Basing on the given data and derived versors, the position of point SIP is determined by its radius vector

$$
\boldsymbol{r}_{S I P}=0,5 l_{0} \hat{\boldsymbol{x}}_{0}+l_{1} \hat{\boldsymbol{q}}_{1}-0,5 l_{2} \hat{\boldsymbol{q}}_{2}+0,5 l_{2}, \hat{\boldsymbol{y}}_{0}+l_{4} \hat{\boldsymbol{q}}_{4}-0,5 l_{6} \hat{\boldsymbol{y}}_{6}+s_{76} \hat{\boldsymbol{z}}_{6}
$$

where: $s_{76}$ - is the variable distance between the seat 7 and platform 6 .

The angular velocity of the platform and the seat $\boldsymbol{\omega}_{6}=\left[\begin{array}{lll}\omega_{6 x_{0}} & \omega_{6 y_{0}} & \omega_{6 z_{0}}\end{array}\right]^{T}$ and linear velocity of the point SIP $\boldsymbol{v}_{S I P}=\left[\begin{array}{lll}v_{S I P x_{0}} & v_{S I P y_{0}} & v_{S I P z_{0}}\end{array}\right]^{T}$ can be represented by a matrix of transformations of velocities of links connected to the drives $\boldsymbol{v}_{w}=\left[\begin{array}{lll}\omega_{12} & \omega_{42} & v_{76}\end{array}\right]^{T}$

$$
\begin{aligned}
& \boldsymbol{\omega}_{6}=\boldsymbol{J}_{\omega} \boldsymbol{v}_{w}, \\
& \boldsymbol{v}_{S I P}=\boldsymbol{J}_{v} \boldsymbol{v}_{w} .
\end{aligned}
$$

In order to derive the matrix of transformations $\boldsymbol{J}_{\omega}$ it is required to differentiate Eqs (3.1) over time. Moreover, the relationships associated with the structure of the investigated mechanism should be duly accounted for 


$$
\begin{cases}\boldsymbol{\omega}_{1}=\omega_{2} \hat{\boldsymbol{y}}_{0}+\omega_{12} \hat{\boldsymbol{y}}_{0} & \\ \boldsymbol{\omega}_{i}=\omega_{i} \hat{\boldsymbol{y}}_{0} & i=2,3 \\ \boldsymbol{\omega}_{j}=\omega_{2} \hat{\boldsymbol{y}}_{0}+\omega_{j 2} \hat{\boldsymbol{x}}_{6} & j=4,5,6\end{cases}
$$

Thus obtained first vector equation is projected onto the directions of versors $\hat{\boldsymbol{x}}_{0}, \hat{\boldsymbol{y}}_{0}$, and the second equation onto the versor directions $\hat{\boldsymbol{x}}_{6}$ and $\hat{\boldsymbol{y}}_{6}$

$$
\left\{\begin{array}{l}
l_{2} \omega_{2}\left(\hat{\boldsymbol{z}}_{0} \cdot \hat{\boldsymbol{q}}_{2}\right)+l_{3} \omega_{3}\left(\hat{\boldsymbol{z}}_{0} \cdot \hat{\boldsymbol{q}}_{3}\right)=l_{1}\left(\omega_{2}+\omega_{12}\right)\left(\hat{\boldsymbol{z}}_{0} \cdot \hat{\boldsymbol{q}}_{1}\right), \\
l_{2} \omega_{2}\left(\hat{\boldsymbol{x}}_{0} \cdot \hat{\boldsymbol{q}}_{2}\right)+l_{3} \omega_{3}\left(\hat{\boldsymbol{x}}_{0} \cdot \hat{\boldsymbol{q}}_{3}\right)=l_{1}\left(\omega_{2}+\omega_{12}\right)\left(\hat{\boldsymbol{x}}_{0} \cdot \hat{\boldsymbol{q}}_{1}\right), \\
l_{5} \omega_{52}\left(\hat{z}_{6} \cdot \hat{\boldsymbol{q}}_{5}\right)=l_{4} \omega_{42}\left(\hat{\boldsymbol{z}}_{6} \cdot \hat{\boldsymbol{q}}_{4}\right), \\
l_{5} \omega_{52}\left(\hat{\boldsymbol{y}}_{6} \cdot \hat{\boldsymbol{q}}_{5}\right)+l_{6} \omega_{62}=l_{4} \omega_{42}\left(\hat{\boldsymbol{y}}_{6} \cdot \hat{\boldsymbol{q}}_{4}\right) .
\end{array}\right.
$$

Solving the system of Eqs (3.8) and recalling Eqs (3.7), we get the elements of the matrix

$$
\boldsymbol{J}_{\omega}=\left[\begin{array}{ccc}
0 & j_{12} & 0 \\
j_{21} & 0 & 0 \\
0 & j_{32} & 0
\end{array}\right] .
$$

Differentiating (3.4) with respect to time, then recalling Eqs (3.7) and projecting it onto the versor directions $\hat{\boldsymbol{x}}_{0}, \hat{\boldsymbol{y}}_{0}, \hat{\boldsymbol{z}}_{0}$ yields the elements of the matrix $\boldsymbol{J}_{v}$.

$$
\boldsymbol{J}_{v}=\left[\begin{array}{ccc}
k_{11} & k_{12} & k_{13} \\
0 & k_{22} & k_{23} \\
k_{31} & j_{32} & k_{33}
\end{array}\right]
$$

Elements of matrices $\boldsymbol{J}_{\omega}, \boldsymbol{J}_{v}$ are dependent on the constant dimensions of the mechanism and on the scalar products of relevant versors.

The real motion platform is actuated by three rotating motors (Fig.1), connected to links 1 and 4 and the platform 6 (Fig.2) via cranks and connectors. The contribution of drives to the platform motion can be expressed by a diagonal matrix $\boldsymbol{J}_{n}$ of transformations of drive velocities $\boldsymbol{\omega}_{n}=\left[\begin{array}{lll}\omega_{n 1} & \omega_{n 2} & \omega_{n 3}\end{array}\right]^{T}$ mapped to velocities of links connected to the drives

$$
\boldsymbol{v}_{w}=\boldsymbol{J}_{n} \boldsymbol{\omega}_{n}
$$

Elements of the matrix $\boldsymbol{J}_{n}$ are derived from the kinematic analysis of four-bar linkages and for the vertical motion - of the slider block linkage (Fig.1). 
It is assumed that considerable loads due to gravity and inertia are associated with the masses of the platform 6, of the seat and the operator. Friction forces are neglected, flexibility of drives and links is taken to be negligible. An assumption is made that the behaviour of springs relieving the vertical motion drive reveals no hysteresis. In the context of these assumptions, the dynamic behaviour of the real motion platform can be governed by the balance equation of instantaneous powers associated with three torque values, the gravity force, the spring force and inertia interactions of the platform 6 , the seat and the operator

$$
\boldsymbol{\omega}_{n}^{T} \boldsymbol{M}_{n}=m_{p} \boldsymbol{v}_{S I P}^{T}\left(\dot{\boldsymbol{v}}_{S I P}-\boldsymbol{g}\right)+\boldsymbol{\omega}_{6}^{T}\left(\boldsymbol{I}_{p} \dot{\boldsymbol{\omega}}_{6}+\tilde{\boldsymbol{\omega}}_{6} \boldsymbol{I}_{p} \boldsymbol{\omega}_{6}\right)-\boldsymbol{v}_{s p}^{T} \boldsymbol{F}_{s p}
$$

where: $\boldsymbol{M}_{n}=\left[\begin{array}{lll}M_{n 1} & M_{n 2} & M_{n 3}\end{array}\right]^{T}$ - the matrix of torques in rotating drives, $v_{s p}=\left[\begin{array}{lll}0 & 0 & v_{s p}\end{array}\right]^{T}$ - the matrix containing the rate of length variations of spring relieving the vertical motion drive; $F_{s p}=\left[\begin{array}{lll}0 & 0 & 2\left(k \Delta s_{s p}+F_{s p_{0}}\right)\end{array}\right]^{T}$ - the matrix taking into account the force of the two springs, $k-$ the elasticity coefficient, $\Delta s_{s p}$ - the spring deflection, $F_{s p_{0}}$ - the initial tension force of the spring.

Substituting Eqs (3.5), (3.6), (3.11) into Eq.(3.12), we obtain

$$
\boldsymbol{\omega}_{n}^{T} \boldsymbol{M}_{n}=m_{p} \boldsymbol{\omega}_{n}^{T} \boldsymbol{J}_{n}^{T} \boldsymbol{J}_{v}^{T}\left(\dot{\boldsymbol{v}}_{S I P}-\boldsymbol{g}\right)+\boldsymbol{\omega}_{n}^{T} \boldsymbol{J}_{n}^{T} \boldsymbol{J}_{\omega}^{T}\left(\boldsymbol{I}_{p} \dot{\boldsymbol{\omega}}_{6}+\tilde{\boldsymbol{\omega}}_{6} \boldsymbol{I}_{p} \boldsymbol{\omega}_{6}\right)-\boldsymbol{\omega}_{n}^{T} \boldsymbol{J}_{n}^{T} \boldsymbol{J}_{s p}^{T} \boldsymbol{F}_{s p}
$$

Equation (3.13) is true as long as the equation below is satisfied

$$
\boldsymbol{M}_{n}=m_{p} \boldsymbol{J}_{n}^{T} \boldsymbol{J}_{v}^{T}\left(\dot{\boldsymbol{v}}_{S I P}-\boldsymbol{g}\right)+\boldsymbol{J}_{n}^{T} \boldsymbol{J}_{\omega}^{T}\left(\boldsymbol{I}_{p} \dot{\boldsymbol{\omega}}_{6}+\tilde{\boldsymbol{\omega}}_{6} \boldsymbol{I}_{p} \boldsymbol{\omega}_{6}\right)-\boldsymbol{J}_{n}^{T} \boldsymbol{J}_{s p}^{T} \boldsymbol{F}_{s p}
$$

where: $\boldsymbol{J}_{s p}=\left[\begin{array}{ccc}0 & 0 & 0 \\ 0 & 0 & 0 \\ 0 & 0 & n_{33}\end{array}\right]$ - the matrix of transformations of seat velocity with respect to the platform 6 mapped to the rate of spring length variations, $\tilde{\boldsymbol{\omega}}_{6}=\left[\begin{array}{ccc}0 & -\omega_{6 z_{0}} & \omega_{6 y_{0}} \\ \omega_{6 z_{0}} & 0 & -\omega_{6 x_{0}} \\ -\omega_{6 y_{0}} & \omega_{6 x_{0}} & 0\end{array}\right], \boldsymbol{I}_{p}-$ inertia matrix, $m_{p}-$ lifted mass.

Equation (3.14) is the basis for determining the critical values of seat vibration frequency and amplitudes for the given asynchronous motors.

\section{Calculation algorithm}

The motion platform is actuated by two asynchronous motors with the power output $1000[\mathrm{~W}]$ and one motor of $750[\mathrm{~W}]$. All motors were connected to identical planetary reduction gears with the gear ratio 1:10. Geometric dimensions and masses are summarised on the basis of the motion platform mechanism design. 


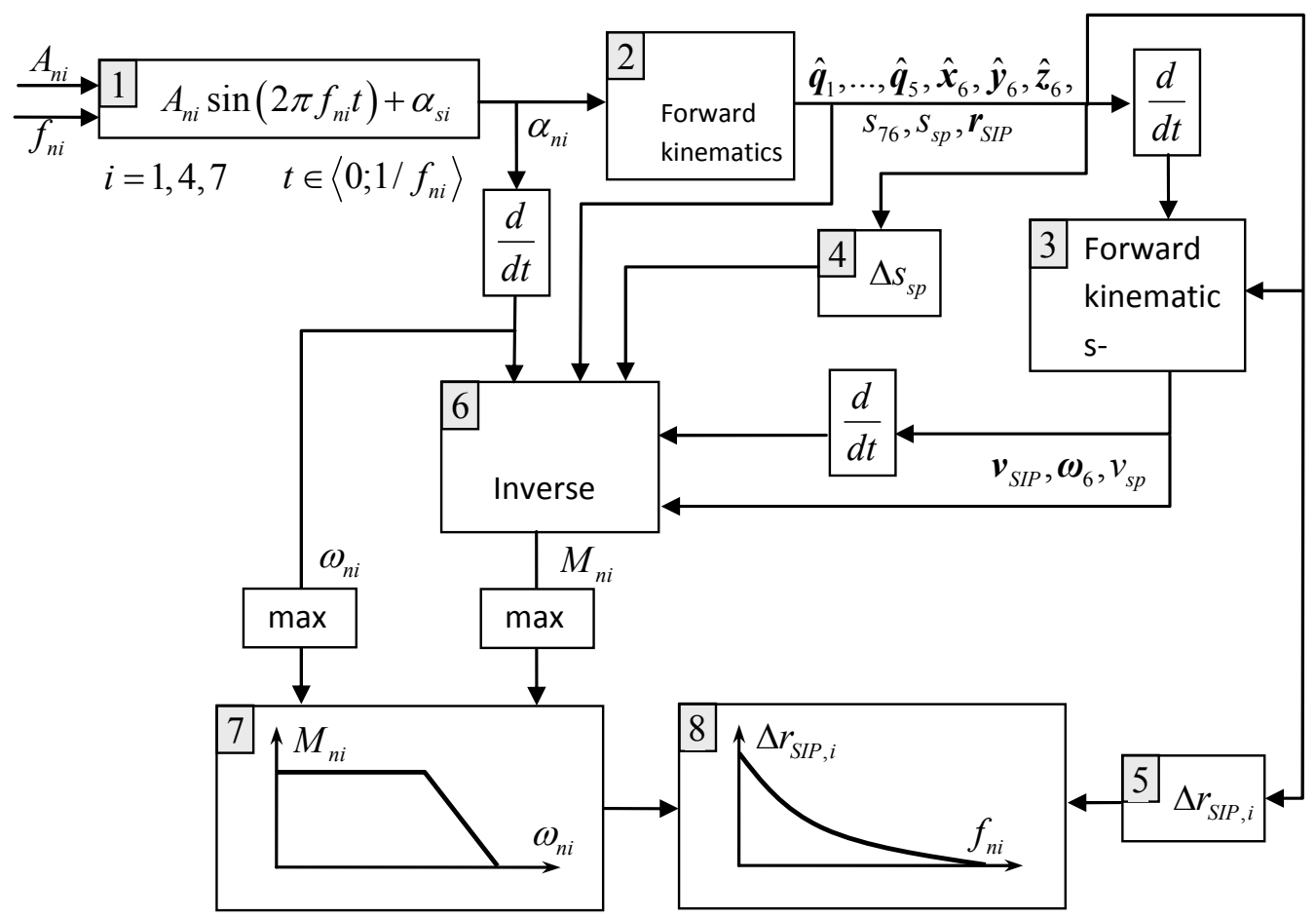

Fig.3. Block diagram of the calculation procedure.

In the first step of the calculation procedure in the block 1 we specify the values of amplitude $A_{n i}$ and frequency $f_{n i}$ of angular motion of the $i$-th drive in the neighbourhood of the initial value $\alpha_{s i}$ (the two remaining drives are immobile). The duration of the simulation procedure is equal to a single period $t \in\left\langle 0,1 / f_{n i}\right\rangle$. In the block 2 the simple problem of positions is solved based on instantaneous values of driving shafts' rotation angles $\alpha_{n i}$ yielding all mobile directional vectors, distances between the seat and the upper platform $s_{76}$, spring lengths $s_{s p}$ and the radius vector of the point SIP- $\boldsymbol{r}_{S I P}$. Velocity of the point SIP, $\boldsymbol{v}_{S I P}$, angular velocity of the seat and the upper platform $\omega_{6}$ and the rate of spring length change $v_{s p}$ are derived in a procedure implemented in block 3 . In relation to thus obtained positions, velocities and accelerations, in block 6 Eq.(3.12) is recalled which enables us to find the instantaneous torque values in the three drives. When the maximal torque and angular velocity values (taking the gears into account) determine a point on the motor characteristic (block 7), then the assumed frequency of the $i$-th drive and the corresponding range of point SIP displacement, computed in block 5, yield the resultant plot (block 8).

\section{Simulation results and conclusions}

The results are expressed as maximal displacement ranges of the point SIP as a function of frequency of motion of a selected drive when the characteristics of the investigated drives reveal the maximal torque or angular velocity values. 


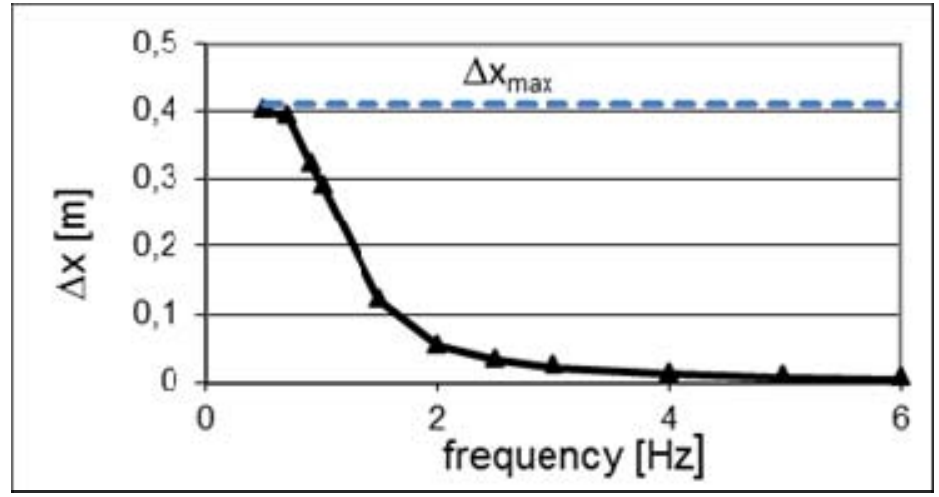

Fig.4. Displacement of the point SIP in the direction of the vehicle's longitudinal axis as a function of frequency of the drive applied to the link 1.

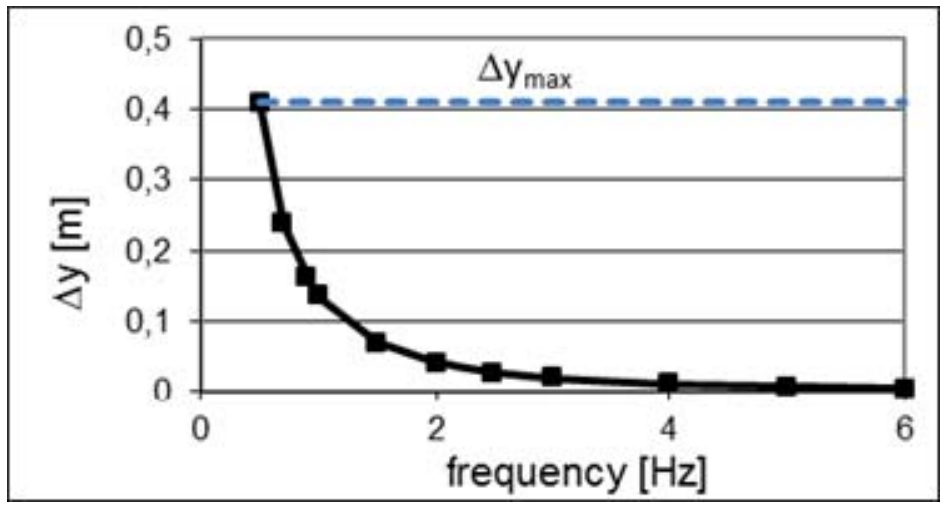

Fig.5. Displacement of the point SIP in the direction of the vehicle's lateral axis as a function of frequency of the drive applied to the link 4.

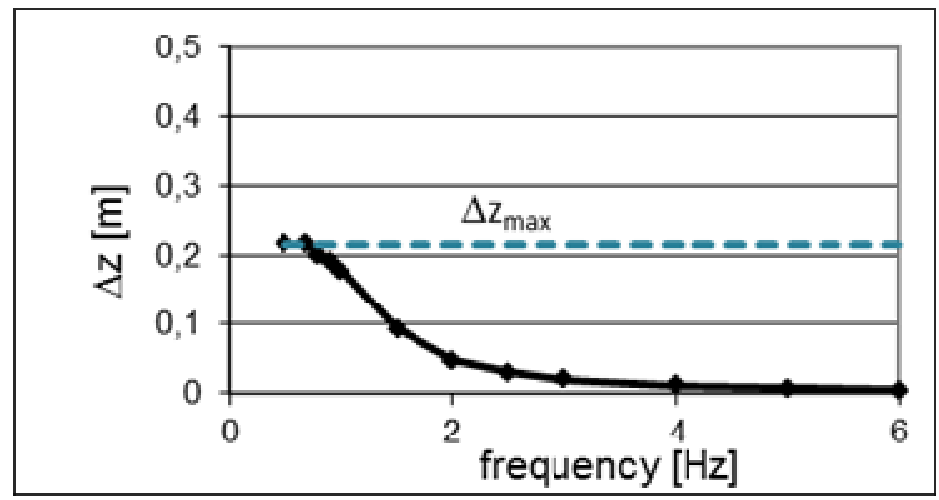

Fig.6. Displacement of the point SIP in the direction of the vehicle's vertical axis as a function of frequency of the drive applied to the seat

Results can be evaluated in relation to measured seat motion data obtained for the wheeled loader Dressta 530R. Figure 7 shows frequency spectra of the seat's acceleration in three investigated directions, the considered frequency range is from 0 to $10 \mathrm{~Hz}$.

Simulations revealed that the applied drives are capable of transmitting loads over the entire displacement range for frequencies below $0.5 \mathrm{~Hz}$. At higher frequencies, the motion range is much lower, and at $6 \mathrm{~Hz}$ it is approximately equal to $5 \mathrm{~mm}$. The frequency analysis of acceleration signals from the loader 
Dressta 530R reveals that the motion is induced up to $6 \mathrm{~Hz}$. An application of springs relieving the vertical motion drive allows the use of a $750 \mathrm{~W}$ motor.

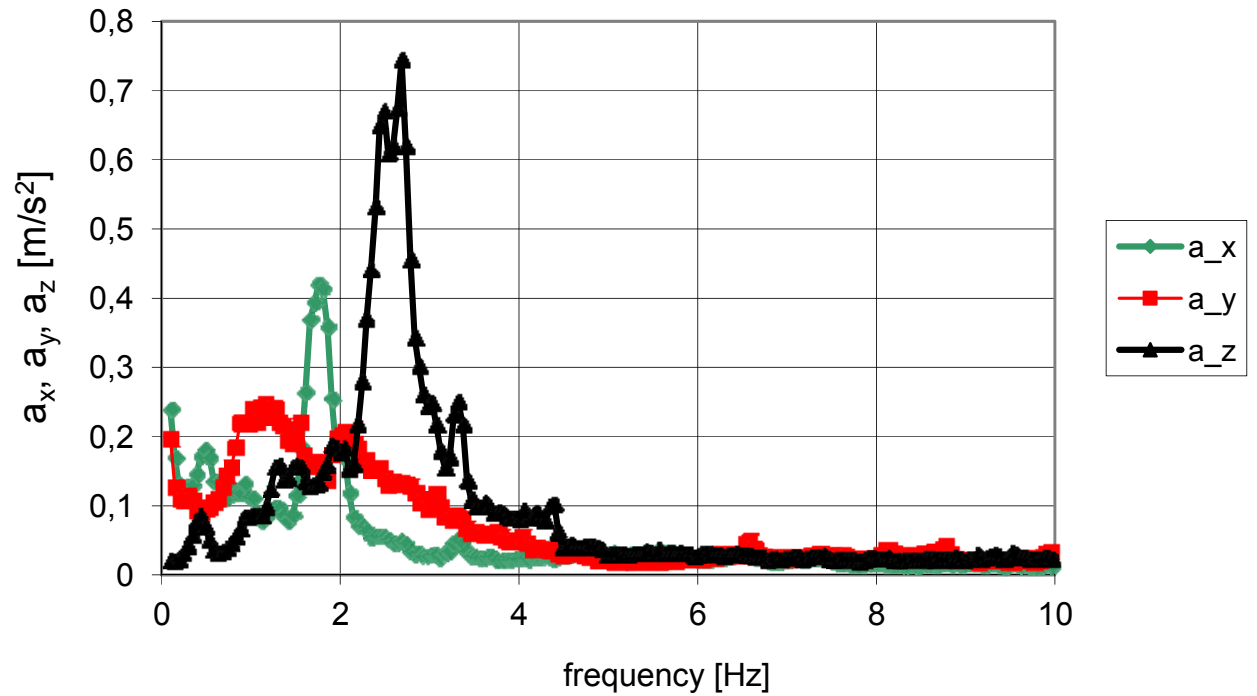

Fig.7. Seat acceleration spectrum in the function of frequency.

\section{References}

[1] Regulation by the Minister of Labour and Social Policy of 6-th June 2014 on the maximal admissible concentrations and intensity of hazardous agents and factors at work, Journal of Laws 817/2014.

[2] Bélanger A., Gagnon S. and Stinchcombe A. (2015): Crash avoidance in response to challenging driving events: The roles of age, serialization, and driving simulator platform. - Accident Analysis and Prevention, vol.82, pp.199212.

[3] Ilka Zöller I., Betz A., Mautes N., Scholz L., Abendroth B., Bruder R. and Winner H. (2015): Valid representation of a highly dynamic collision avoidance scenario in a driving simulator. - Transportation Research Part F 31, pp.54-66.

[4] Helland A., Jenssen G.D., Lervåg L-E., Westin A.A., Moen T., Sakshaug K., Lydersen S., Mørland J. and Lars Slørdal L. (2013): Comparison of driving simulator performance with real driving after alcohol intake: A randomised, single blind, placebo-controlled, cross-over trial. - Accident Analysis and Prevention, vol.53, pp.9-16.

[5] Sadeghi Tehran D., Nahvi A., Hajirasouli M., Naseri H., Kh. Lotfi Kh. and Niknejad M. (2016): Effects of stimulant and opiate drugs on driver behavior during lane change in a driving simulator. - Travel Behaviour and Society 4, pp.69-78.

[6] Lozia Z. (2008): Vehicle Ride Simulators. - Warsaw: WKŁ.

[7] Feng Gao, Weimin Li, Xianchao Zhao, Zhenlin Jin and Hui Zhao (2002): New kinematic structures for 2-, 3-, 4-, and 5-DOF parallel manipulator designs. - Mechanism and Machine Theory, vol.37, pp.1395-1411.

[8] Gogu G. (2008): Structural Synthesis of Parallel Robots, Part 1 - Methodology. - Dordrecht: Springer.

[9] Tora G. (2012): Modelling of a platform mechanism of an active suspension system in a heavy machine. - Series: Mechanics, Monograph 416, Cracow University of Technology, Cracow.

[10] Casas S., Alcaraz J.M., Olanda R., Inmaculada Coma I. and Fernández M. (2014): Towards an extensible simulator of real motion platforms. - Simulation Modelling Practice and Theory, vol.45, pp.50-61.

[11] Uicker J.J., Pennock Jr. G.R. and Shigley J.E. (2011): Theory of Machines and Mechanisms. - New York: Oxford, Oxford University Press. 